\title{
Evaluation of Efficacy of Various Implants in Maxillary Arch Distalization - A finite Element Anolusis
}

\author{
Dr Sneha Sanap,' Dr Vinit Swami, ${ }^{2}$ Dr Amol Patil,3 Dr Shailesh Deshmukh,4 Dr Veera Bhosales \\ Post-Graduate Student, $2-4$ Professor, 5 Professor \& Head
} Department of Orthodontics, Bharati Vidyapeeth Deemed to be University Dental College and Hospital, Pune, India.

Correspondence: Dr Sneha Sanap; Email: sneha.sanap7@gmail.com

\section{ABSTRACT}

Introduction: Orthodontic correction of Angle's class II molar relation has, for long, been one of the challenge in orthodontics, with various researchers attempting to correct the class II molar relationship by diverse methods. One of the technique that has gained popularity in recent times is maxillary arch distalization by infrazygomatic screws and miniscrews. The objective of the study is to measure and compare the amount of maxillary arch distalization and its effects, on adjacent teeth, by varying the positions of mini-implants by Finite Element Analysis.

Materials \& Method: A standard three-dimensional finite element model was constructed to simulate the maxillary teeth, periodontal ligament, and alveolar process. In this study, three models were prepared. Model-1: The (miniscrews) were placed between upper first and second premolar, and between second premolar and first molar bilaterally. Model-2: Infrazygomatic screws was placed between upper first and second molar bilaterally. Model-3: Infrazygomatic screws was placed on the mesiobuccal root of upper first molar bilaterally. The displacement of each tooth was calculated on $x, y$, and $z$ axes when $200 \mathrm{gm}$ of force was applied on each side.

Result: Maximum amount of maxillary arch distalization was seen when infrazygomatic screws placed between upper first and second molar in model-2. Whereas maximum amount of maxillary arch intrusion and less distalization was observed when miniscrews placed between upper first premolar and second premolar and in between second premolar and upper first molar in model-1. The difference was statistically significant $\left(p=0.005^{*}\right)$. There was no bucco-palatal rotation of teeth observed among all three finite element models.

Conclusion: Thus infrazygomatic screws and miniscrews are the effective means of maxillary arch distalization for the correction of Class II malocclusion.

Keywords: Class II malocclusion; Finite element; Maxillary arch distalization; Miniscrews.

\section{INTRODUCTION}

Maxillary arch distalization is an increasingly popular option to correct the class II malocclusion with nonextraction approach. Headgearhas been conventional modality for class II malocclusion through distalization of molars or entire the maxillary dentition. However, its main disadvantage is patient compliance. To avoid this drawback, various non-compliance appliances have been developed including Keles slider, repelling magnets, distal jet, and pendulum. These devices applied continuous forces to distalize maxillary arch, which might lead to distal tipping and extrusion of the first molars and mesial reactive forces might cause anchorage loss and labial flaring of anterior teeth. ${ }^{\prime}$

To overcome unwanted side-effects, recent technique such as maxillary arch distalization by mini-implants are more effective and give good results. Miniscrews were introduced in clinical orthodontics for the purpose of orthodontic anchorage, and these presented the clinician with a versatile option. ${ }^{2-7}$ Sugawara et. al. (2006) proposed maxillary dentition distalization by using titanium anchor plates. ${ }^{8}$ Miniscrew implants provides absolute anchorage and their ability to retract whole dentitions can eliminate adverse reciprocal movement and maximize the efficiency of the treatment. ' In addition, miniscrews provides the option, for early loading after placement which reduces treatment time.

The application of monocortical miniscrew type temporary anchorage devices to various clinical situations demanding movement of either a single 
tooth or teeth segment has been largely successful, with the ease and minimal invasiveness at insertion and removal..$^{10}$ The miniscrews placed at the interdental alveolar bone can deliver forces directly to the tooth or archwire, eliminating the need for additional connectors. This versatility of the miniscrews can be very helpful, especially for the posterior segment control, for which extraoral appliances used to be indicated. ${ }^{11,12}$

Additional miniscrews in the premolar area appear to facilitate intrusion and distalization of the entire arch according to the position of the force vectors. A new method for distalization of the entire maxillary dentition is using miniscrews implanted in the infrazygomatic crest, as proposed by Liou et. al. ${ }^{13}$ and Lin and Liou. ${ }^{14}$ They suggested that upper first and upper second molar region is the most ideal safe zone for placing miniscrews in the buccal alveolar bone in the infrazygomatic crest region for maxillary dentition distalization.

Estimation of precise stresses of distalization on the maxillary arch, periodontal ligament and alveolar bone is difficult in in-vivo studies. The stress generated in the periodontal ligament and tooth root can be more accurately studied with the help of an in-vitro numerical finite element model. Finite element analysis was introduced into Orthodontics by Yettram et. al. (1977) in 1972.15

The FEM is an engineering resource used to calculate stress and deformations in complex structures, and it has been widely applied in biomedical research. ${ }^{16}$ In FEM, 3D models allow better understanding of the mechanical and structural behaviour of the dental tissues and structures, providing more realistic and accurate results resembling the actual occurrence in in-vivo studies as recommended by Tajima et.al. (2009)..$^{17}$

Hence, this study was undertaken to evaluate the efficacy and effect of various (mini) implants position in maxillary arch distalization by Finite Element Analysis.

\section{MATERIALS AND METHOD}

This study was conducted to evaluate the efficacy of various implants (miniscrews) in maxillary arch distalization and also to measure and compare the amount of distalization and its effects on adjacent structures with Finite Element Analysis. This study is approved by Institutional Research Committee.

\section{Finite Element Model}

Computer configuration used for the study:

i. Hardware: A computer (DELL XPS System) with Intel i7 with 8-core processor, 8 GB RAM, 2 GB Graphics, loaded with Windows 7 operating system was used.

ii. Software: Three types of softwares were used in finite element analysis for pre-processing, FEA solver and post-processing. They were Altair HyperMesh, Altair OptiStruct and Altair Hyperworks (Altair Engineering Inc., Troy, Michigan, USA) respectively.

The titanium miniscrews and infrazygomatic crest (IZC) screw were modeled. The miniscrews used were $7 \mathrm{~mm}$ in length, $1.8 \mathrm{~mm}$ in coronal diameter and tapered body (Dentos India Pvt. Ltd.). The dimension of IZC screws were $8 \mathrm{~mm}$ in length, $2 \mathrm{~mm}$ in diameter. The main archwire was modeled according to the dimension of a $0.016 " \times 0.022$ " stainless steel archwire. Niti-coil spring of wire diameter $0.25 \mathrm{~mm}$ and lumen size 0.049" was used.

The finite element model was constructed by scanning normal human maxillary jaw with alveolar bone and teeth obtained from scanning centre. The geometric model of standard MBT prescription brackets and tubes (0.018"x 0.025" slot), Stainless Steel (SS) wire (0.016" x $0.022 ")$, miniscrews, IZC, Niti-coil spring and crimpable hook was also constructed (Fig 1).

In this study, three finite element models were prepared.

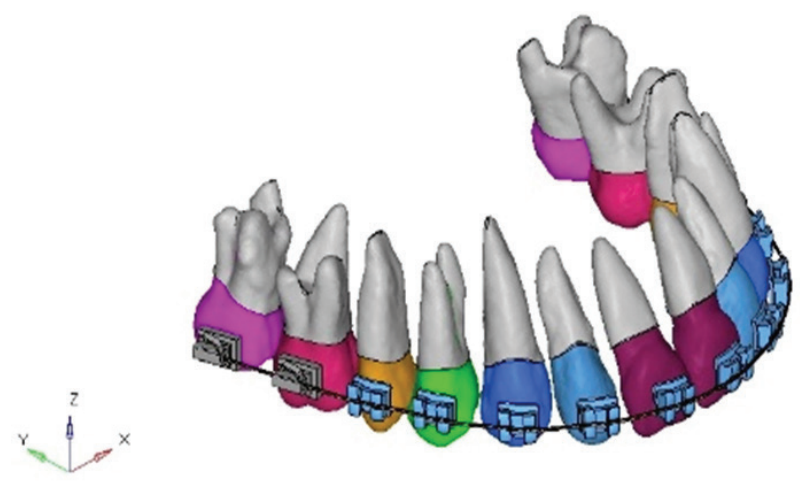

Figure 1: FEM model with (MBT prescription 0.018 "x0.025" slot) brackets and 0.016"x0.022" stainless steel wire. 


\section{Force application}

\section{Model-1}

The miniscrews were placed bilaterally between maxillary first and second premolar and between second premolar and first molar at the height of $5 \mathrm{~mm}$ from alveolar crest 9 (Fig 2). Miniscrews were inserted at the midpoint between adjacent teeth with $45^{\circ}$ angulations relative to the occlusal plane and on the mucogingival junction. The crimpable hooks of height $4 \mathrm{~mm}$ were attached between lateral incisor and canine and between canine and first premolar on $0.016 \times 0.22$ SS on each side. The Niti-coil springs from each miniscrew head to corresponding crimpable hooks with a force magnitude of approximately 200 gm on each side were attached for maxillary arch distalization (Fig 5).

\section{Model-2}

The titanium IZC screws were placed bilaterally between maxillary first molar and second molar

Model 1

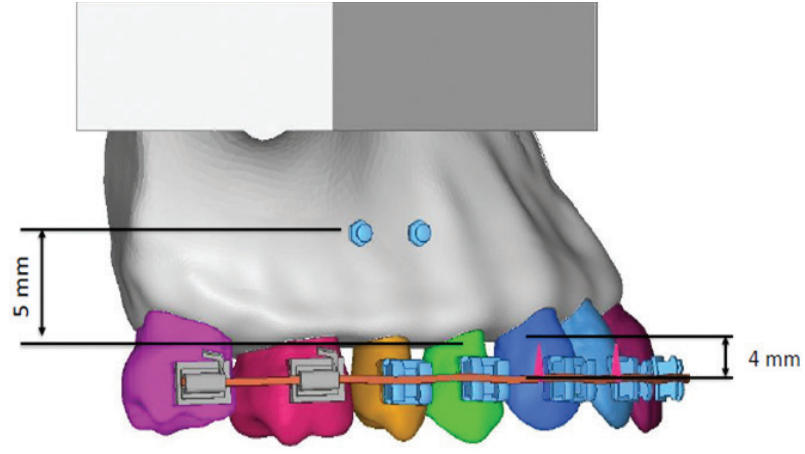

Figure 2: Miniscrews placed between maxillary first and second premolar and between maxillary second premolar and first molar at the height of $5 \mathrm{~mm}$ from alveolar crest.

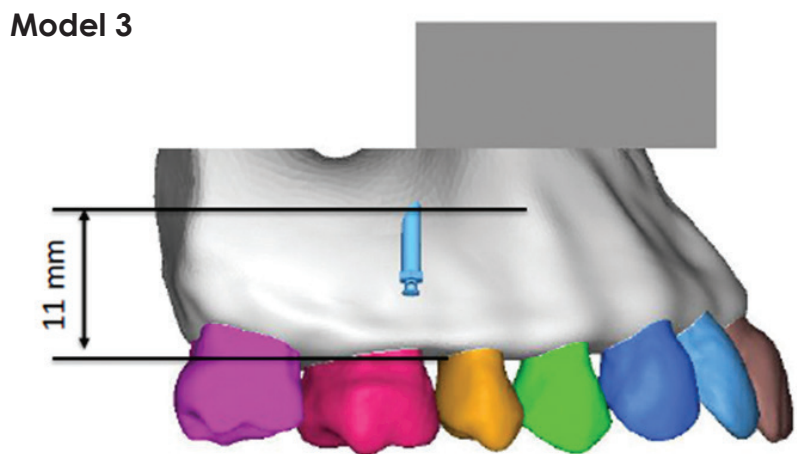

Figure 4: Infrazygomatic crest screw placed on the mesio-buccal root of maxillary first molar at the height of $11 \mathrm{~mm}$ from alveolar crest. on the buccal side of root at an angle of $55-70^{\circ}$ to maxillary occlusal plane at height of $11 \mathrm{~mm}$ from alveolar crest 9(Fig 3). The force of $200 \mathrm{gm}$ on each side was applied from Niti- coil spring for maxillary arch distalization (Fig 6)

\section{Model-3}

The titanium IZC screws were placed bilaterally on the mesio-buccal root of maxillary first molar on the buccal side at an angle of $55-70^{\circ}$ to maxillary occlusal plane at height of $11 \mathrm{~mm}$ from alveolar crest 9(Fig 4). The force of $200 \mathrm{gm}$ on each side was applied from Niti- coil spring for maxillary arch distalization (Fig 7).

The material properties like density, Young's modulus and Poisson's ratio of various components which were used in the study in order to simulate the actual properties of the components are shown in Table 1.

The meshing details of various components used in the study are shown in table 2.

\section{Model 2}

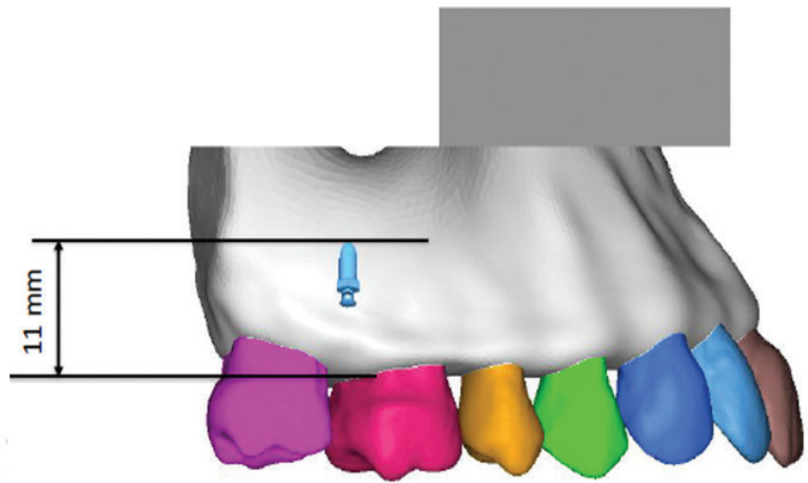

Figure 3: Infrazygomatic crest screw placed between maxillary first and second molar at the height of 11 $\mathrm{mm}$ from alveolar crest.

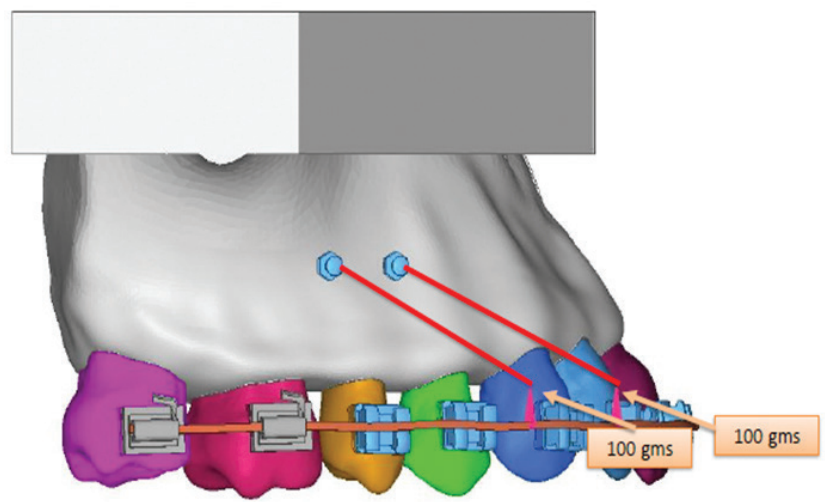

Figure 5: $200 \mathrm{gm}$ of force was applied from miniscrews to archwire through Niti- coil spring. 


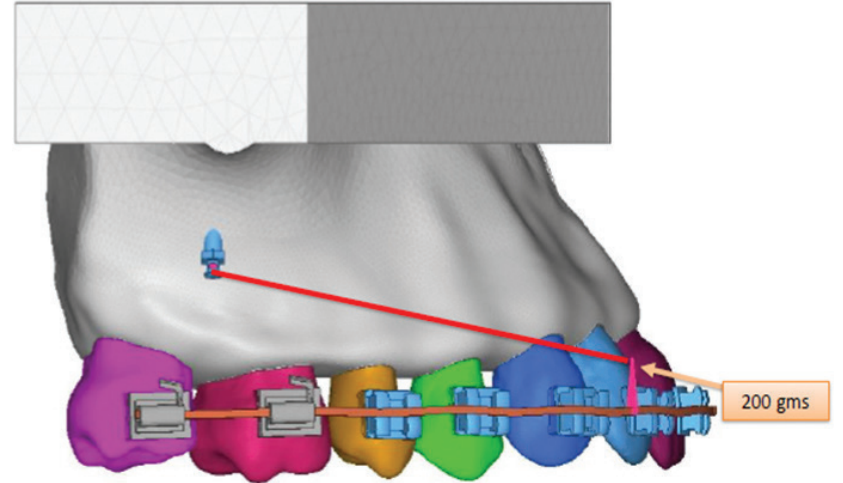

Figure 6: $200 \mathrm{gm}$ of force was applied from infrazygomatic crest screw to archwire through Niticoil spring.

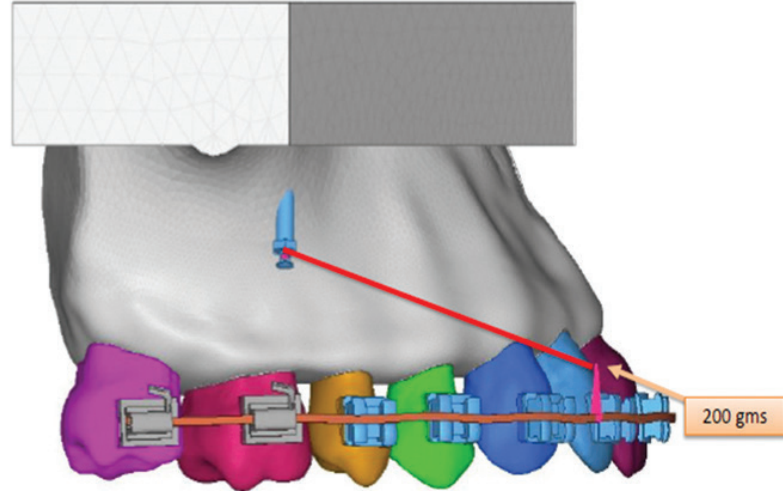

Figure 7: $200 \mathrm{gm}$ of force was applied from infrazygomatic crest screw placed on mesio-buccal root to archwire through Niti- coil spring.

Table 1: Material properties of teeth, periodontal ligament, alveolar bone, stainless steel and titanium

\begin{tabular}{|l|c|c|c|}
\hline \multicolumn{1}{|c|}{ Component } & Density $\left(\mathbf{g} / \mathbf{m m}^{\wedge} \mathbf{3}\right)$ & Young Modulus $(\mathbf{M P a})$ & Poisson's Ratio $(\boldsymbol{\mu})$ \\
\hline Teeth & $1.7 \mathrm{E}-06$ & $2.03 \mathrm{E}+04$ & 0.3 \\
\hline Periodontal ligament (PDL) & $1.7 \mathrm{E}-06$ & 0.667 & 0.49 \\
\hline Alveolar bone & $1.7 \mathrm{E}-06$ & $1.37 \mathrm{E}+04$ & 0.38 \\
\hline Stainless Steel & 0.008 & $2.1 \mathrm{E}+05$ & 0.3 \\
\hline Titanium & $4.4 \mathrm{E}-03$ & $1.1 \mathrm{E}+05$ & 0.342 \\
\hline
\end{tabular}

Table 2: Meshing details of teeth, periodontal ligament, alveolar bone, cortical bone, brackets, titanium miniscrews and archwire.

\begin{tabular}{|l|c|c|}
\hline \multicolumn{1}{|c|}{ Component } & No. of Nodes & No. of elements \\
\hline Teeth & 26954 & 123722 \\
\hline Periodontal Ligament (PDL) & 38952 & 63365 \\
\hline Alveolar bone & 29877 & 92102 \\
\hline Cortical bone & 15675 & 43765 \\
\hline Brackets & 2776 & 8927 \\
\hline Titanium Mini Screw & 6263 & 12326 \\
\hline Arch wire & 2241 & 992 \\
\hline
\end{tabular}

\section{Statistical analysis}

SPSS for Windows, Version 16.0. Chicago, SPSS Inc software was used to analyse the data. Statistical analysis was done by using tools of descriptive statistics such as Mean, and SD for representing quantitative data. The level of significance was set at $5 \%$. Hence, $\mathrm{p}$ value less than 0.05 was termed as significant. Confidence interval set at $95 \%$, Power of the study at $80 \%$ were set while calculating sample size. Data normality was checked by Shapiro-Wilk test. For skewed data, non-parametric tests like Kruskal-Wallis test, Mann-Whitney $U$ test were applied. Kruskal-Wallis test was used to determine the statistical difference of mean amount of distalization, intrusion and buccopalatal rotation values between the three models. Mann-Whitney test was used to determine the statistical difference of mean amount of distalization, intrusion and bucco-palatal rotation values of teeth between multiple individual pair wise group comparisons.

\section{RESULT}

All the results were tabulated and also shown in the form of graphs to visualize the statistical difference more clearly. Mean and standard deviations, for the millimetric readings of the study were measured and compared. The results of this study were obtained from simulated models, hence biologic variabilities may occur. All values were multiplied by $1 \mathrm{E}+4$ to arrive at simple numerical values for comparison \& analysis purpose.

The highest amount of arch distalization was observed in model 2 as compared to model 1 and 3. On overall 
Table 3: Measurement and comparison of maxillary arch distalization among all FEM models

\begin{tabular}{|c|c|c|c|c|}
\hline DISTALIZATION & Mean $(\mathrm{mm})$ & S.D & Kruskal- Wallis 'H' test & $\mathrm{p}$ value, Significance \\
\hline Model 1 & 0.768 & 0.505 & \multirow{3}{*}{$H=2.523$} & \multirow{3}{*}{$p=0.283$} \\
\hline Model 2 & 1.199 & 0.904 & & \\
\hline Model 3 & 1.068 & 0.740 & & \\
\hline \multicolumn{5}{|c|}{ Mann- Whitney test to find pair wise individual comparison } \\
\hline Group & Comparison Group & Mean Difference & \multicolumn{2}{|c|}{ p value, Significance } \\
\hline \multirow{2}{*}{ MODEL 1} & MODEL 2 & 0.431 & \multicolumn{2}{|c|}{$p=0.279$} \\
\hline & MODEL 3 & 0.300 & \multicolumn{2}{|c|}{$p=0.532$} \\
\hline MODEL 2 & MODEL 3 & 0.131 & \multicolumn{2}{|c|}{$p=0.885$} \\
\hline
\end{tabular}

$p>0.05$ - no significant difference, ${ }^{*} p<0.05$ - significant difference, ${ }^{* *} p<0.001$ - highly significant difference

Table 4: Measurement and comparison of intrusion in maxillary arch distalization among all FEM models.

\begin{tabular}{|c|c|c|c|c|}
\hline INTRUSION & Mean $(\mathrm{mm})$ & S.D & Kruskal- Wallis 'H' test & p value, Significance \\
\hline Model 1 & 0.624 & 0.432 & \multirow{3}{*}{$H=1.746$} & \multirow{3}{*}{$p=0.003^{*}$} \\
\hline Model 2 & 0.158 & 0.223 & & \\
\hline Model 3 & 0.223 & 0.241 & & \\
\hline \multicolumn{5}{|c|}{ Mann- Whitney test to find pair wise individual comparison } \\
\hline Group & Comparison Group & Mean Difference & \multicolumn{2}{|c|}{ p value, Significance } \\
\hline \multirow{2}{*}{ MODEL 1} & MODEL 2 & 0.465 & \multicolumn{2}{|c|}{$\mathrm{p}=0.001^{*}$} \\
\hline & MODEL 3 & 0.400 & \multicolumn{2}{|c|}{$p=0.005^{*}$} \\
\hline MODEL 2 & MODEL 3 & 0.064 & \multicolumn{2}{|c|}{$p=0.849$} \\
\hline
\end{tabular}

$p>0.05$ - no significant difference, ${ }^{*} p<0.05$ - significant difference, ${ }^{* *} p<0.001$ - highly significant difference

Table 5: Measurement and comparison of bucco-palatal rotation in maxillary arch distalization among all FEM models

\begin{tabular}{|c|c|c|c|c|}
\hline Bucco-palatal rotation & Mean $(\mathrm{mm})$ & S.D & Kruskal- Wallis 'H' test & $p$ value, Significance \\
\hline Model 1 & 0.091 & 0.100 & \multirow{3}{*}{$\mathrm{H}=0.137$} & \multirow{3}{*}{$p=0.934$} \\
\hline Model 2 & 0.084 & 0.060 & & \\
\hline Model 3 & 0.081 & 0.057 & & \\
\hline \multicolumn{5}{|c|}{ Mann- Whitney test to find pair wise individual comparison } \\
\hline Group & Comparison Group & Mean Difference & \multicolumn{2}{|c|}{ p value, Significance } \\
\hline \multirow{2}{*}{ MODEL 1} & MODEL 2 & 0.006 & \multicolumn{2}{|c|}{$p=0.972$} \\
\hline & MODEL 3 & 0.009 & \multicolumn{2}{|c|}{$p=0.938$} \\
\hline MODEL 2 & MODEL 3 & 0.003 & \multicolumn{2}{|c|}{$p=0.993$} \\
\hline
\end{tabular}

$p>0.05$ - no significant difference, ${ }^{*} p<0.05$ - significant difference, ${ }^{* *} p<0.001$ - highly significant difference

comparison of distalization among three models using Kruskal- Wallis ' $\mathrm{H}$ ' test, there were no significant difference ( $p>0.05)$ among the groups. On using Mann-Whitney test, no statistical significant difference was found ( $p>0.05$ ) among all FEM models (Table 3).

The highest amount of arch intrusion was observed in model 1 as compared to model 2 and 3. On overall comparison of intrusion among three models using Kruskal- Wallis ' $\mathrm{H}$ ' test, significant difference was found ( $p>0.05$ ) among the groups. On using Mann- Whitney test to find pair wise individual comparison, it was found that Model 2 had lesser intrusion of arch value, as compared to model 1 and the difference was statistically significant $\left(p=0.001^{*}\right)$. Model 3 also showed lesser intrusion value as compared to model 1 and the difference was statistically significant $\left(p=0.005^{*}\right)$. Model 2 had lesser mean intrusion of maxillary arch value as compared to Model 3 but the difference was not statistically significant ( $p>0.05)$ (Table 4).

On overall comparison of rotation among three models using Kruskal-Wallis ' $H$ ' test and by using Mann-Whitney test to find pair wise individual comparison, there were no statistical significant difference ( $p>0.05)$ among all FEM models (Table 5).

The individualised effect on teeth's during maxillary arch distalization has been explained in (Fig 8). A standard coordinate system was constructed with the 

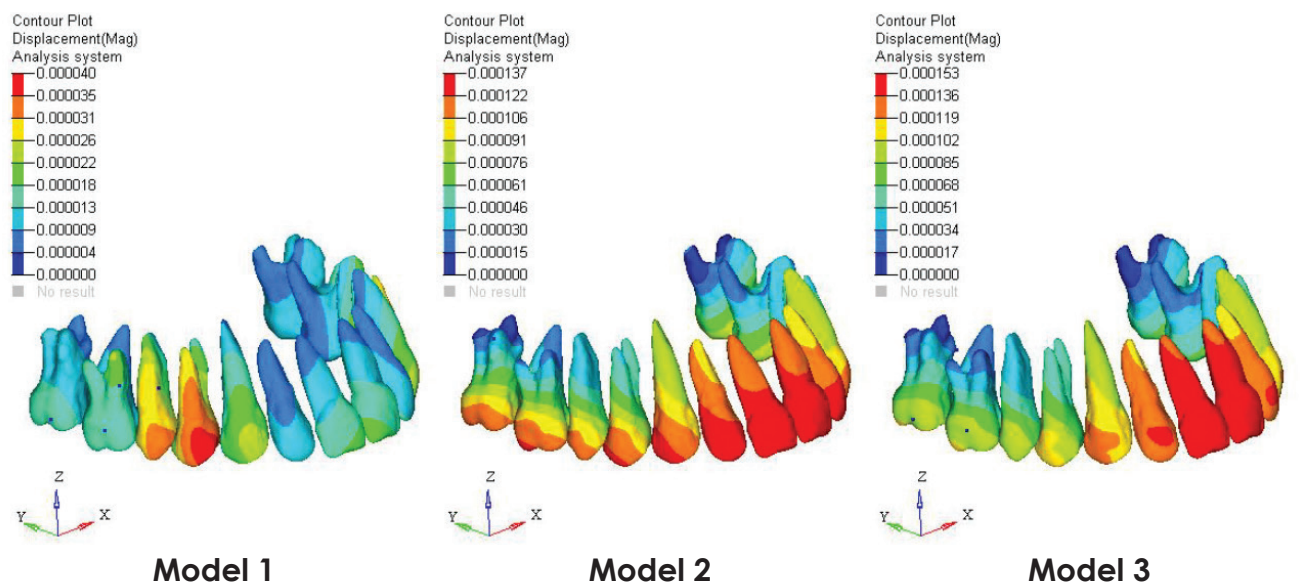

Model 2

Model 3

Figure 8 (A): Displacement of maxillary arch in Y-Direction (Distalization).

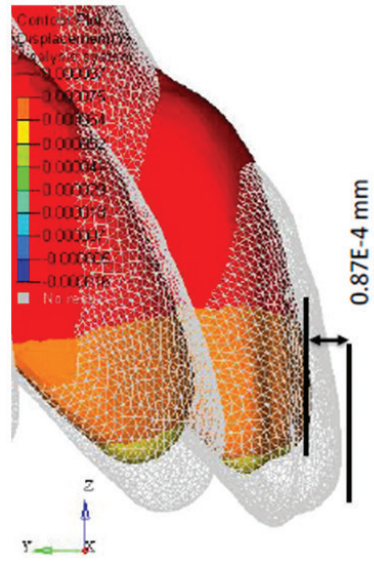

Model 1

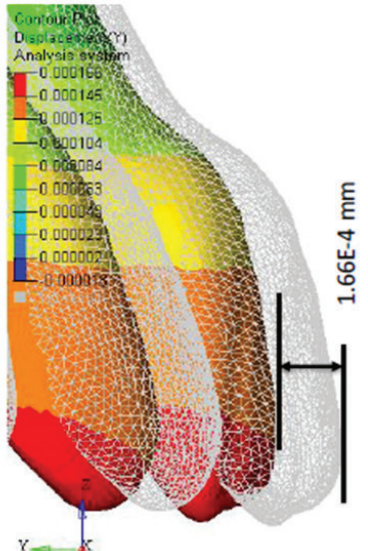

Model 2

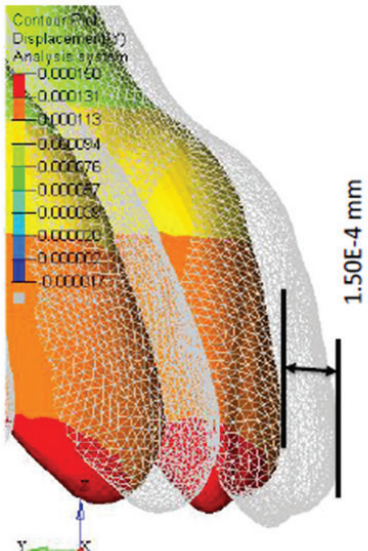

Model 3

Figure 8 (B): Amount of distalization of maxillary teeth (Anterior segment).

$x$-axis corresponding to the bucco-palatal direction, the $y$-axis the antero-posterior direction, and the z-axis the superior-inferior direction.

The central and lateral incisors were moved distally by $0.6 \mathrm{~mm}$, and canines by $0.7 \mathrm{~mm}$. The first and second premolars were distalized by $0.6 \mathrm{~mm}$. The first and second molars were distalized by $2.1 \mathrm{~mm}$ bilaterally, in model 1. (Fig 8.A).

The central and lateral incisors were moved distally by $1.5 \mathrm{~mm}$, and canines by $1.5 \mathrm{~mm}$. The first and second premolars were distalized by $1.3 \mathrm{~mm}$. The first and second molars were distalized by $2.9 \mathrm{~mm}$ bilaterally, in model 2.

The central and lateral incisor were moved distally by $1.3 \mathrm{~mm}$, and canine by $1.3 \mathrm{~mm}$. The first and second premolar were distalized by $1.2 \mathrm{~mm}$. The first and second molar were distalized by $2.5 \mathrm{~mm}$ bilaterally, in model 3
Maximum amount of maxillary arch distalization was seen in model 2, i.e. when IZC screw placed between upper first and second molar because of more horizontal vector, as compared to other (mini) implants position. This states from graph 1.

The central and lateral incisors were intruded by 1.2 $\mathrm{mm}$ and $1.1 \mathrm{~mm}$ respectively. Canines were intruded by $0.9 \mathrm{~mm}$. Minimum intrusion was seen at first and second premolar, $0.5 \mathrm{~mm}$ and $0.3 \mathrm{~mm}$ respectively. Least intrusion was seen in posterior region. The first and second molars were intruded by $0.1 \mathrm{~mm}$, bilaterally in model 1 (Fig 9.A).

The central and lateral incisors were intruded by 0.06 $\mathrm{mm}$ and $0.03 \mathrm{~mm}$ respectively. Very least intrusion was seen for canines, $0.006 \mathrm{~mm}$. The first and second premolars were intruded by $0.045 \mathrm{~mm}$ and $0.002 \mathrm{~mm}$ respectively. The first and second molars were intruded by $0.018 \mathrm{~mm}$ and $0.040 \mathrm{~mm}$ respectively, in model 2 . 
Table No.- 3: Tooth Displacement in Y-Axis

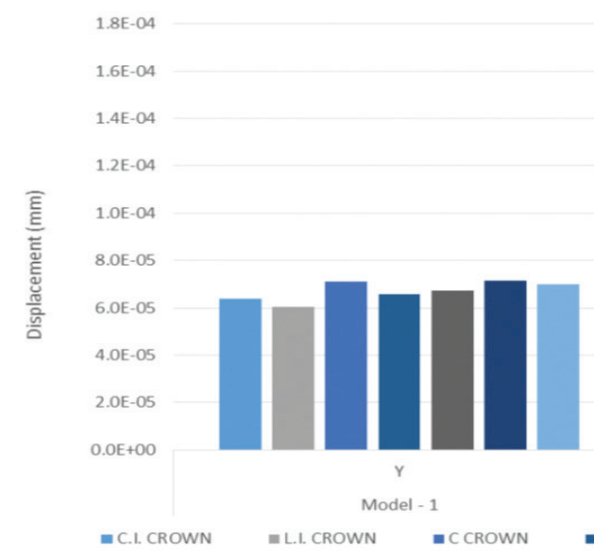

Model 1

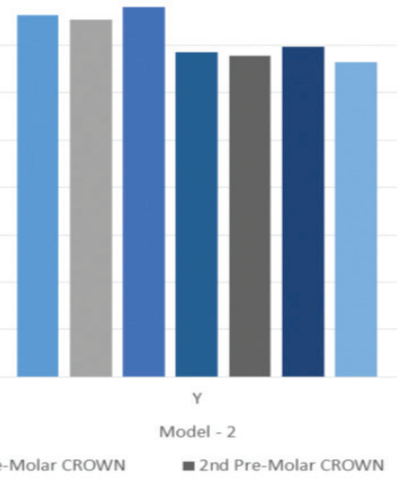

Model 2

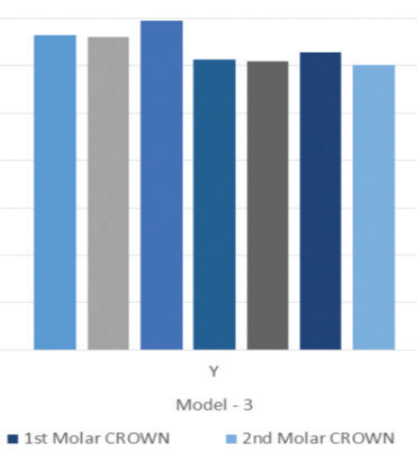

Model 3

Graph 1 showing tooth displacement in distalization direction (Y-axis)

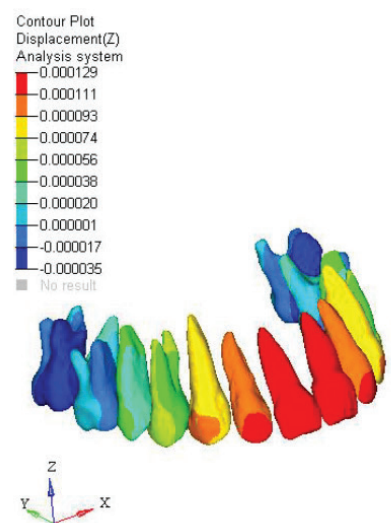

Model 1

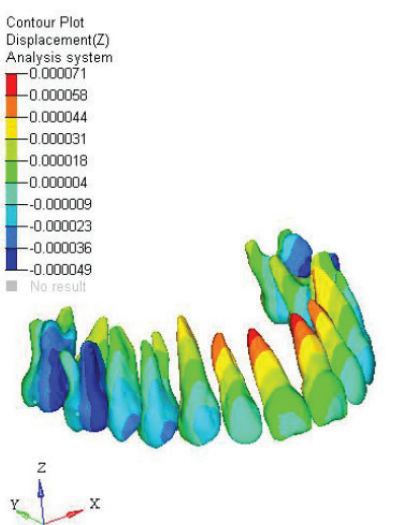

Model 2

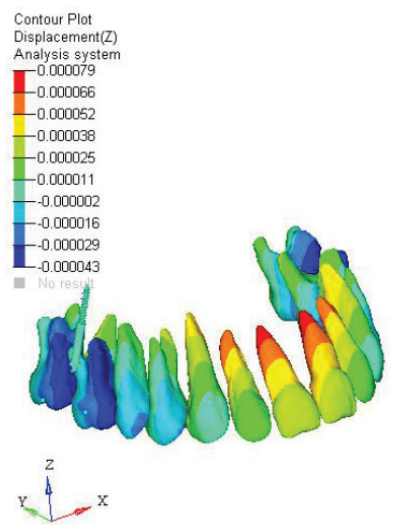

Model 3

Figure 9 (A): Displacement of maxillary arch in Z-Direction (Intrusion).

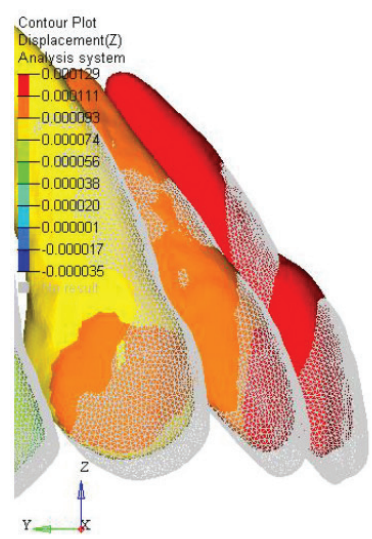

Model 1

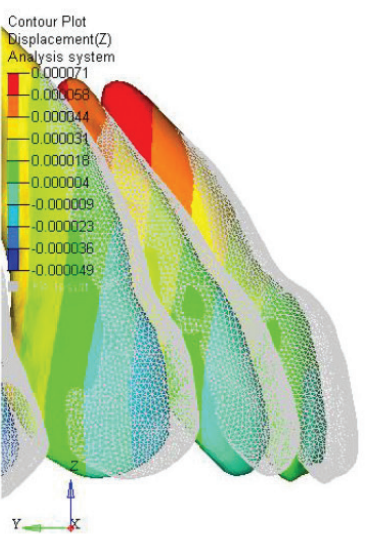

Model 2

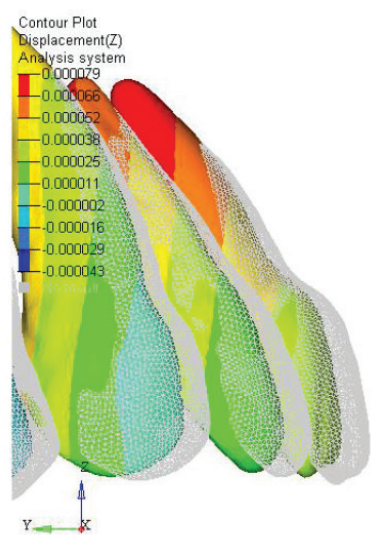

Model 3

Figure 9 (B): Amount of intrusion of maxillary teeth (Anterior segment). 


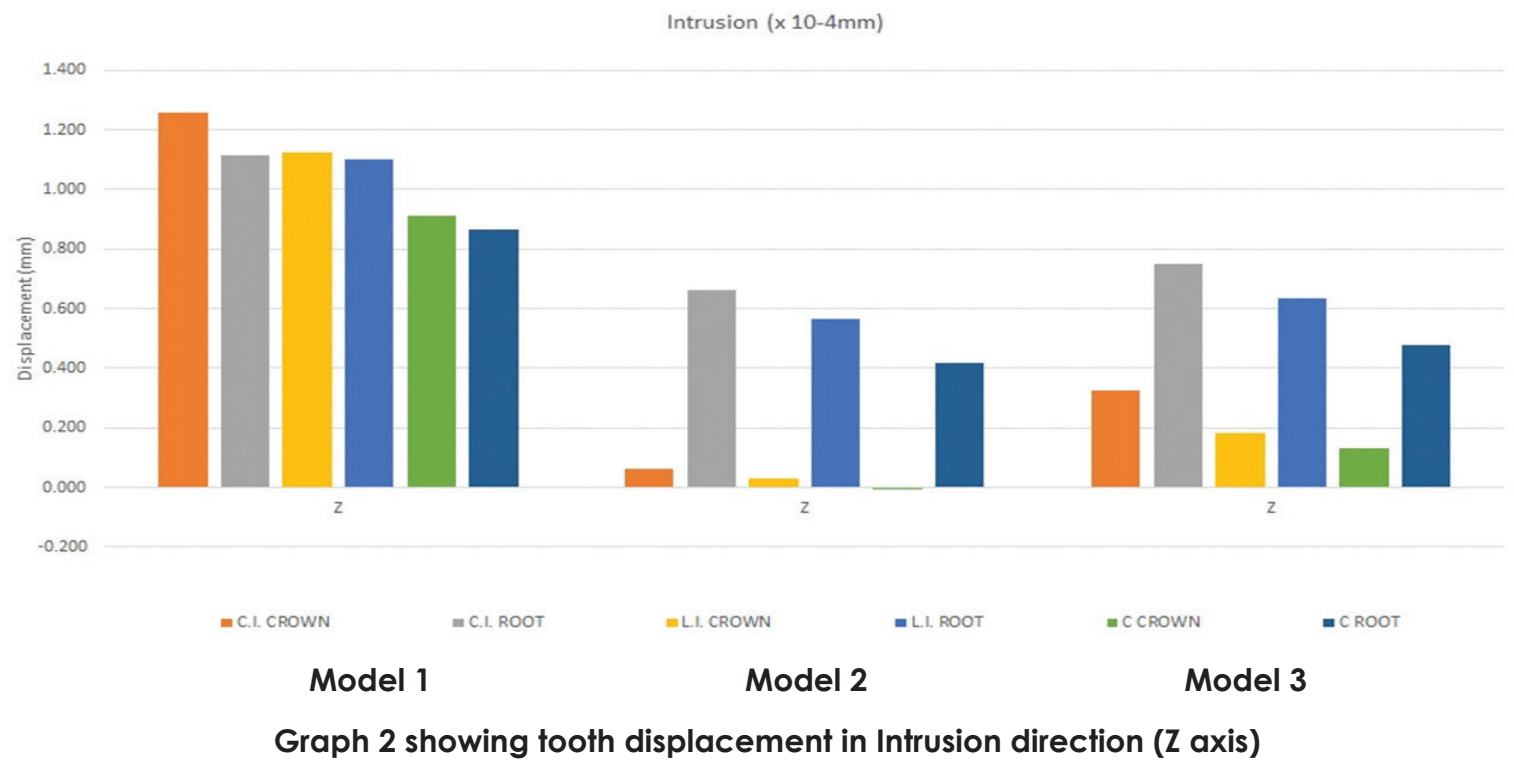

The central and lateral incisors were intruded by $0.3 \mathrm{~mm}$ and $0.1 \mathrm{~mm}$ respectively. The canines were intruded by $0.1 \mathrm{~mm}$. The first and second premolars were intruded by 0.1 and $0.04 \mathrm{~mm}$ respectively. The first and second molars were intruded by $0.008 \mathrm{~mm}$ in model 3 .

Maximum intrusion was seen on the anteriors in maxillary arch distalization in model 1 i.e. when (mini) implants were placed between first and second premolar and between second premolar and first molar, as compared to IZC screw. (Fig 9.B). This states from graph 2.

This result shows that maximum amount of maxillary arch distalization and least intrusion were seen when IZC screws placed between upper first and second molar. Whereas maximum amount of maxillary arch intrusion and less distalization was observed when miniscrews placed between upper first and second premolar and in between second premolar and first molar. There was no bucco-palatal rotation of teeth observed among all three FEM model.

\section{DISCUSSION}

Effective correction of Angle's class II molar relation has, for long, been one of the enigmas of orthodontics, with various researchers attempting to correct the class II molar relationship by diverse methods. One of the technique that has gained popularity in recent times is maxillary arch distalization. Numerous designs for arch distalization appliances have been reported in the literature including the Hilgers pendulum appliance, the Cetlin headgear, removable appliance and open nickel-titanium (NiTi) push coils. Most of these devices suffer from a loss of anterior anchorage and relapse after removal of the distalization appliance. ${ }^{18-22}$

Orthodontic treatment with miniscrew anchorage is more comfortable for the patient than traditional reinforced anchorage such as multi-brackets combined with intraoral or extraoral anchorage, because there is no requirement for the patient's cooperation. The success rate of miniscrews is approximately $80-95 \%$, and minimum invasion for placement surgery is necessary; the patients complained of little pain and discomfort after placement of the miniscrews.

The center of resistance (Cres) of the maxillary dentition has been shown to be located around the middle area of the premolar roots.23The more interproximal alveolar bone is available between the maxillary second premolar and first molar roots and between the maxillary first and second molar roots than in other locations. ${ }^{21}$

The three dimensional FEM model used in the study provides the freedom to simulate the orthodontic force system applied clinically and allows analysis in the response of the dentition to the orthodontic load in three dimensional spaces. ${ }^{24}$

This study was undertaken to evaluate the efficacy of various (mini) implants position in maxillary arch distalization by Finite Element Analysis. This study was performed with the help of Finite Element 
Method (FEM), which examines the biomechanical characteristics of orthodontic tooth movement. FEM offers an ideal method for accurate modeling of the tooth-periodontium system with its complicated threedimensional geometry.

Sung et. al. (2015) evaluated stress distribution and displacement patterns of the entire maxillary arch with regard to distalizing force vectors applied from interdental miniscrews by finite element analysis. In his study, FE model was prepared by using around 65507 elements and 2106 nodes for PDL. ${ }^{25}$ Whereas in the present study, 63365 elements and 38952 nodes for PDL were used to provide more accurate result.

Several studies have been done on the amount of force required for maxillary arch distalization. In the present study same $200 \mathrm{gm}$ of force was applied on both sides for distalization. ${ }^{23,26,27}$

Distalizing force was applied through Niti-coil spring to the retraction hook between lateral incisor and canine, the amount of force generated with full compression of coil spring was about $200 \mathrm{gm}$. This force system would allow application of consistent force at the level of the center of resistance of maxillary dentition, located around the middle area of premolar roots. ${ }^{28}$ Displacement of the teeth was found for all models.

Liou et. al. (2007) and Lin and Liou (2003) proposed a new method for distalization of the entire maxillary dentition by using miniscrews implanted in the infrazygomatic crest. They suggested that upper first and second molar region is the most ideal safe zone for placing miniscrews in the buccal alveolar bone in the infrazygomatic crest region for maxillary dentition distalization.

Liou et. al. (2007) recommended placing a miniscrew at the mucogingival junction for dentition distalization, because adequate buccal thickness of the alveolar bone was necessary. The $5-\mathrm{mm}$ plane from the alveolar crest edge was taken as the initial plane for the measurement. The thickest buccal alveolar bone is located in the U6-U7 region above the $5-\mathrm{mm}$ plane which is $3.55 \mathrm{~mm}$. Similar height of miniscrews is used in the present study. Currently, the diameters of most miniscrews are 1.2-2 mm.29.30 Since a minimum of $1 \mathrm{~mm}$ of alveolar bone around the screw could be sufficient for periodontal health, considering bone thickness and miniscrew strength, the diameter of miniscrew should be $2 \mathrm{~mm}^{31}$ Laursen et. al. (2013) also reported that perpendicular insertion at the midroot level only rarely interfered with the sinus, whereas apically inclined insertion increased the risk of sinus perforation. They also suggested that safe zone for miniscrew insertion is between maxillary first and second molar for whole arch distalization. ${ }^{32}$

Our study shows maximum amount of distalization on each side when IZC is placed between upper first and second molar, as compared to other two implants positions. Incisors show minimum intrusion of $0.18 \mathrm{~mm}$ and distalization of $3 \mathrm{~mm}$ on each side. Molars were distalized by $5.8 \mathrm{~mm}$ on each side and show bodily movement. When distalizing force is applied to an anteriorly located hook, movement of the total arch is effectively induced because of more horizontal force vector. This finding is almost similar to result obtained clinically by Choi et. al. (2011) where molars were distalized by $4.5 \mathrm{~mm}$ on right side and $3.5 \mathrm{~mm}$ on left side, but incisors were intruded and retracted by $1 \mathrm{~mm}$ on both sides, ${ }^{26}$ which is not similar to present study, because of less vertical force vector.

In the present study, distalizing force is applied to an anteriorly located hook, between lateral incisor and canine and the height of retraction hook is $4 \mathrm{~mm}$ from arch wire which shows greater distal movement of whole maxillary arch, which is similar to sung et.al. (2015) 25 study.

When IZC were placed on the mesio-buccal root of upper first molar on both the sides, shows minimum amount of whole arch distalization as compared to other implant position. In this, incisors were intruded and distalized by $0.4 \mathrm{~mm}$ and $1.3 \mathrm{~mm}$ respectively on each side. Molars were distalized by $5 \mathrm{~mm}$ on each side. This finding is concordant to result obtained clinically by Xiaove et.al.(2018) where molars were distalized by 4.5 $\mathrm{mm}{ }^{33}$

Rodrigues et.al. (2018) placed IZC screw on distobuccal root of upper first molar on both the sides for maxillary arch distalization. In this, incisors were intruded by $2.5 \mathrm{~mm}$ and molars were distalized by $3.7 \mathrm{~mm} .{ }^{34}$ The is good option for maxillary arch distalization.

The present study shows least amount of maxillary arch distalization when miniscrews were placed in between upper first and second premolar and in between second premolar and first molar, but more intrusion 
of anterior segment was observed because the force vector is less horizontal, as compared to other implant position. Incisors were intruded and distalized by 2.3 $\mathrm{mm}$ and $1.2 \mathrm{~mm}$ respectively on each side. Molars were distalized by $4.2 \mathrm{~mm}$ on each side. This finding is not similar to result obtained clinically by Bechtold et. al. (2013). In his study, incisors were intruded by $1.4 \mathrm{~mm}$ and molars were distalized by $2.9 \mathrm{~mm}$ and $1.83 \mathrm{~mm}$ on both sides when dual and single miniscrews were placed respectively. ${ }^{23}$ Park et. al. (2015) also studied maxillary arch distalization by placing implants in same position as in present study, but the results are not similar. Incisors were intruded by $1.4 \mathrm{~mm}$ and molars were distalized by $2 \mathrm{~mm} .{ }^{35}$ Though the force vectors are same, they applied power chains instead of Niticoil spring, as power chain shows force degradation, so the intrusion and distalization was less as compared to our study.

Kuroda et. al. (2007) found failures near the end of maxillary arch distalization, possibly due to the roots of the teeth might come in contact with miniscrews. ${ }^{36}$ To avoid this failures, Liou et. al. suggested that, replacement was made by placing the new miniscrew in the same interradicular area with 2-mm clearance from the initial insertion sites for the continuity of treatment. Special attention should always be given to positioning the implants far from the roots of the anterior teeth, not only to present an initial clearance of $2 \mathrm{~mm}$, but also to present a safe distance of 7 to $10 \mathrm{~mm}$ between the miniscrew implants and the roots of the adjacent teeth, which is necessary for the retraction of the anterior teeth after the distalization. ${ }^{37}$

\section{CONCLUSION}

1. Maximum distalization was seen when IZC screw placed between upper first and second molar as compared to other two position (i.e. miniscrews placed between upper first and second premolar and between upper second premolar and first molar, and IZC screw placed on the mesio-buccal root of upper first molar). But no statistical significant difference was found.

2. Maximum amount of intrusion was seen of anterior segment when miniscrews placed between upper first and second premolar and between upper second premolar and first molar as compared to IZC screws. The results were statistically significant.

3. No bucco-palatal rotation was seen by varying positions of implant in all FEM models.

Thus by using IZC and miniscrews in maxillary arch distalization is an effective way, for the correction of class II malocclusion.

\section{Limitations}

1. Analytical results of FEM are highly dependent on the models developed; therefore, they have to be constructed to be equivalent to real objects in various aspects. The results of this study were obtained from simulated models, from which biologic variabilities may occur.

2. Finite element can only calculate initial tooth displacement after force application. The biological and time-dependent reaction is still unpredictable and requires more clinical evidence.

\section{Conflicts of interest}

There are no conflicts of interest. 


\section{REFERENCES}

1. Yu II, Kook YA, Sung SJ, Lee KJ, Chun YA, Sung SM. Comparison of tooth displacement between buccal mini-implants and palatal plate anchorage for molar distalization: a finite element study. Eur J Orthod 2014;36(4):394-442.

2. Creekmore TD, Eklund MK. The possibility of skeletal anchorage. J Clin Orthod 1983; 17(4):266-269.

3. Kanomi R. Mini-implant for orthodontic anchorage. J Clin Orthod 1997;31 (11):763-767.

4. Costa A, Raffaini M, Melsen B. Miniscrews as orthodontic anchorage: a preliminary report. Int J Adult Orthod Orthogn Surg 1998; 13(3) :201-209.

5. Park HS. The Use of Micro-Implant as Orthodontic Anchorage. Seoul, South Korea: Nare Pub Co.2001;83-151.

6. Park HS, Bae SM, Kyung HM, Sung JH. Micro-implant anchorage for treatment of skeletal Class I bialveolar protrusion. J Clin Orthod 2001; 35(7):417-422.

7. Park HS, Kyung HM, Sung JH. A simple method of molar uprighting with micro-implant anchorage. J Clin Orthod 2002; 36(10):592-596.

8. Sugawara J, Kanzaki R, Takahashi I, Nagasaka H, Nanda R. Distal movement of maxillary molars in nongrowing patients with the skeletal anchorage system. Am J Orthod Dentofac Orthop 2006; 129:723-733.

9. Liu H, Wu X, Yang L, Ding Y. Safe zones for miniscrews in maxillary dentition distalization assessed with cone-beam computed tomography. Am J Orthod Dentofac Orthop 2017;151: 500-506.

10. Lee KJ, Park YC, Hwang WS, Seong EH. Uprighting mandibular second molars with direct miniscrew anchorage. J Clin Orthod 2007: $41: 627-705$.

11. Park YC, Lee SY, Kim DH, Jee SH. Intrusion of posterior teeth using mini-screw implants. Am J Orthod Dentofac Orthop 2003; 123:690-694.

12. Park HS, Lee SK, Kwon OW. Group distal movement of teeth using microscrew implant anchorage. Angle Orthod 2005; 75:602-609.

13. Liou EJ, Chen PH, Wang YC, Lin JC. A computed tomographic image study on the thickness of the infrazygomatic crest of the maxilla and its clinical implications for miniscrew insertion. Am J Orthod Dentofac Orthop 2007; 131:352-356.

14. Lin JC, Liou EJ. A new bone screw for orthodontic anchorage. J Clin Orthod 2003; 37:676-682.

15. Yettram AL, Wright KW, Houston WJ. Centre of rotation of a maxillary central incisor under orthodontic loading. Br J Orthod 1977 ; 4: $23-27$.

16. Sameshima GT, Melnick M. Finite element-based cephalometric analysis. Angle Orthod 1994;64(5):343-350.

17. Tajima K, Chen K, Takahashi N, Noda N, Nagamatsu Y, Kakigawa H. Three Dimensional finite element modelling from CT images of tooth and its validation. Dent Mater J 2009; 8: 219-226.

18. Cetlin NM, Hoeve T. A nonextraction treatment. J Clin Orthod 1983; 17:396-413.

19. Jones RD, White MJ. Rapid Class III molar correction with open coil jig. J Clin Orthod 1992; 26:661-664.

20. Gianelly AA, BednarJ, Dietz V S. Japenes. Ni-Ti coils used to move molar distally. Am J Orthod Dentofac Orthop 1991; 99:564-566.

21. Jeon JM, YU HS, Baik HS, Lee JS. En-masse distalization with miniscrew anchorage in class II nonextraction treatment. J Clin Orthod 2006: 40:472-476.

22. Hilgers JJ. The pendulum appliance for Class II noncompliance therapy. J Clin Orthod 1992; 26:706-714.

23. Bechtold TE, Kim JW, Choi TH, Park YC. Distalization pattern of the maxillary arch depending on the number of orthodontic miniscrews. Angle Orthod 2013; 83:266-273.

24. Penedo ND, Elias CN, Christina M, Pacheco T, de Gouvêa JP. 3D simulation of orthodontic tooth movement. Dental Press J Orthod 2010; 15:98-108.

25. Sung EH, Kim SJ, Chun YS, Park YC. Distalization pattern of whole maxillary dentition according to force application points. Korean $J$ Orthod 2015;45(1):20-28.

26. Choi YJ, Lee JS, Cha JY, Park YC. Total distalization of the maxillary arch in a patient with skeletal Class II malocclusion. Am J Orthod Dentofac Orthop 2011; 139:823-833.

27. Deshmukh SV, Vadera KJ. Nonextraction treatment with en-masse distalization of maxillary dentition using miniscrews. J Indian Orthod Soc 2018; 52:204-209

28. Keles A, Ervedi N, Sezen S. Bodily distalization of molars with absolute anchorage. Angle Orthod 2003;73(4): 471-482.

29. Maino BG, Mura P, Bednar J. Miniscrew implants: the spider screw anchorage system. Semin Orthod 2005; 11:40-46.

30. Herman R, Cope JB. Miniscrew implants: IMTEC mini ortho implants. Sem Orthod 2005; 11:32-39.

31. Poggio PM, Incorvati C, Velo S, Carano A. "Safe zones": A guide for miniscrew positioning in the maxillary and mandibular arch. Angle Orthod 2006; 76:191-197.

32. Laursen MG, Melsen B, Cattaneo PM. An evaluation of insertion sites for mini-implants: a micro-C T study of human autopsy material. Angle Orthod 2013; 83:222-229.

33. WU X, Liu H, Luo C. Three- dimensional evaluation on the effect of maxillary dentition distalization with miniscrews implanted in the infrazygomatic crest. Implant Dent.2018; 27(1): 22-27.

34. de Almeida MR, de Almeida RR, Nanda R. Biomechanics of extra alveolar mini-implant use in the infrazygomatic crest area for asymmetrical correction of class II subdivision malocclusion. APOS Trends Orthod 2018; 8:110-118.

35. Park HS, Kwon TG, Sung JH. Nonextraction Treatment with Microscrew Implants. Angle Orthod 2004;74(4): 539-549.

36. Kuroda S, Yamada K, Deguchi T. Root proximity is a major factor for screw failure in orthodontic anchorage. Am J Orthod Dentofac Orthop 2007;131: 68-73.

37. Liou EJ, Pai BC, Lin JC. Do miniscrews remain stationary under orthodontic forces? Am J Orthod Dentofac Orthop 2004;126: $42-47$. 